\title{
Una metodologia per calcolare indici di rischio quantitativi dalle richieste di risarcimento delle aziende ospedaliere
}

\author{
Simona Dalle Carbonare ${ }^{1,2}$, Fulvia Folli ${ }^{3}$, Emanuele Patrini ${ }^{4}$, Riccardo Bellazzi ${ }^{1}$ \\ Dipartimento di Informatica e Sistemistica, Università di Pavia \\ Istituto Universitario di Studi Superiori (IUSS), Pavia \\ 3 Risk Manager, Azienda Ospedaliera della Provincia di Lodi \\ 4 Healthcare Risk Manager, MARSH S.p.A., Milano
}

\begin{abstract}
In the last years, the need of preventing medical injuries associated to clinical practice has pushed towards the implementation of risk management strategies in clinical environments. At the same time, the insurance costs that Health Care Organizations (HCOs) have to pay for the coverage of medical injuries steadily increased. Compensation claims for medical injuries are related both to clinical adverse events and to monetary compensations, so they can be exploited to analyze the HCOs risk areas. To this end we developed a probabilistic method, based on an approach developed in financial risk management, to obtain quantitative risk indexes from medical injuries compensation claims. This method was successfully applied to the analysis of the compensation claims data of the Azienda Ospedaliera of Lodi allowing to better understand the risk structure of the Azienda Ospedaliera and of its departments.
\end{abstract}

Keywords: risk management, medical injuries, Monte Carlo method, actuarial analysis, probabilistic models

A methodology for computing quantitative risk indexes from medical injuries compensation claims Pratica Medica \& Aspetti Legali 2010; 4(1): 37-43

\section{INTRODUZIONE}

Negli ultimi anni la prevenzione degli errori, degli incidenti e degli eventi avversi nella pratica clinica è diventata un tema di grande interesse sia per i cittadini, sempre più informati ed esigenti, sia per le aziende ospedaliere, che devono far fronte a premi assicurativi in continuo aumento. Per questo motivo si stanno affermando anche in ambito sanitario tecniche e metodologie mirate alla gestione del rischio (risk management) già consolidate in altre realtà, come quella finanziaria, o in altri sistemi sanitari, quale ad esempio quello britannico.

Uno dei concetti basilari del risk management in sanità è imparare dagli errori e dagli eventi avversi che si sono verificati per capirne le cause ed evitarli in futuro. Il primo passo per l'applicazione di queste tecniche prevede quindi la raccolta e l'analisi dei dati riguardanti gli errori e gli eventi avversi nella pratica medica. I dati relativi agli errori sono però spesso difficilmente reperibili poiché strettamente legati alle responsabilità del singolo e poiché non sono ancora diffusi sistemi di raccolta standardizzati e organizzati a livello nazionale. I dati riguardanti i sinistri invece sono già raccolti dalle aziende ospedaliere o dalle compagnie assicuratrici e possono fornire spunti e risultati interessanti per l'analisi e la gestione del rischio in sanità. I sinistri sono infatti eventi avversi a cui è seguita una richiesta di risarcimento; per questo motivo i dati relativi forniscono informazioni sugli eventi avversi che li hanno provocati e sull'impatto economico associato. Queste informazioni possono essere sfruttate quindi sia nell'ambito della gestione del rischio, sia per una migliore gestione delle politiche assicurative. 
Negli ultimi anni si è verificato inoltre un incremento delle azioni giudiziarie nei confronti degli operatori sanitari e delle strutture ospedaliere dovuto alla maggior consapevolezza dei cittadini che chiedono una sanità sempre più sicura a fronte di un aumento della complessità dei trattamenti. Come conseguenza di questo fenomeno anche i premi assicurativi sono in continua crescita e incidono in maniera sempre più rilevante e preoccupante sul bilancio delle aziende ospedaliere. Il "Rapporto sulla $1^{\circ}$ rilevazione nazionale relativa agli aspetti assicurativi in ambito di gestione aziendale del rischio clinico" [1] conferma questo aumento dei premi assicurativi e rivela un atteggiamento prevalentemente passivo delle aziende ospedaliere nei confronti delle compagnie assicuratrici che calcolano i premi sulla base del monte stipendi senza considerare in maniera rilevante l'effettiva rischiosità della struttura. Il rapporto mostra però che un numero crescente di strutture si sta dotando di un'unità di gestione del rischio clinico e di un'unità operativa per la gestione delle polizze assicurative.

In questo contesto fornire alle aziende ospedaliere uno strumento per poter analizzare la propria rischiosità in maniera quantitativa, anche dal punto di vista economico, può permettere una migliore gestione sia del rischio clinico, sia delle politiche assicurative. Per questo si propone un metodo probabilistico, basato sul modello attuariale [2-4], che permette di analizzare i dati riguardanti le richieste di risarcimento pervenute alle aziende ospedaliere e di ricavare una stima della perdita associata da cui calcolare alcuni indici di rischio quantitativi. La metodologia è stata pensata per poter essere applicata sia alla totalità dei dati, ottenendo un'analisi "globale", sia ai dati raggruppati in base ai valori di variabili di interesse, come la specialità clinica o il centro ospedaliero, effettuando delle analisi "stratificate". Nel contesto in esame l'analisi globale può essere sfruttata per valutare la rischiosità complessiva dell'azienda e gestire in maniera più consapevole le politiche assicurative. Le analisi stratificate possono invece essere impiegate per quantificare e confrontare le rischiosità di singoli settori permettendo di individuare quelli che necessitano di interventi migliorativi.

\section{IL METODO}

Come descritto nell'introduzione, in questo articolo viene introdotto un metodo probabilistico basato sul modello attuariale per stimare la distribuzione della perdita e ottenere indici di rischio quantitativi dai dati relativi alle richieste di risarcimento pervenute alle aziende ospedaliere. Il modello attuariale è largamente diffuso in ambi- to finanziario e bancario, dove le tecniche di risk management sono sfruttate per stimare e gestire il rischio operativo. Il modello permette di ricavare la distribuzione di probabilità della perdita e tra gli indici di maggiore interesse, il Value at risk (Var) $[5,6]$. Il Var può essere definito come la massima perdita attesa, una volta stabiliti:

- l'orizzonte temporale su cui viene valutato, ad esempio quello annuale;

- il livello di confidenza con cui si effettua la stima, solitamente pari al 99\%;

- la valuta associata, nel nostro caso l'euro, particolarmente rilevante se si sta lavorando in un contesto internazionale.

Il modello attuariale calcola il Var come il $99^{\circ}$ percentile della distribuzione della perdita, cioè come il valore della perdita che non viene superato nel 99\% dei casi. Applicando il modello attuariale la perdita viene stimata considerando sia la gravità dell'evento, o severity, sia la sua frequenza di accadimento, o frequency. In questo modo nella stima della perdita sono tenuti in considerazione sia eventi che accadono spesso, ma hanno conseguenze poco gravi, sia eventi rari, ma associati a perdite ingenti. Il metodo proposto prevede dunque di stimare dai dati a disposizione una distribuzione di probabilità per la frequency e una per la severity e di ricavare la distribuzione della perdita applicando una simulazione di tipo Monte Carlo [7-9]. Utilizzando questa tecnica la distribuzione della perdita è ottenuta tramite la generazione di una serie di scenari ipotetici basati sulle distribuzioni di frequency e severity stimate. Ciascuno scenario viene quindi ottenuto seguendo il seguente procedimento, illustrato anche in Figura 1:

1. si estrae un valore di frequency secondo la distribuzione stimata, ad esempio supponiamo di estrarre il valore 2 ;

2. si estrae dalla distribuzione della severity un numero di valori corrispondenti alla frequency estratta, cioè nel nostro esempio si considera un caso ipotetico in cui si verificano 2 eventi che presentano rispettivamente una severity pari a $500 €$ e $1.000 €$;

3. si calcola la perdita corrispondente allo scenario simulato come somma dei valori di severity estratti, ottenendo quindi una perdita pari a $1.500 €$ per il caso considerato.

Generando molti scenari ipotetici, cioè ripetendo molte volte il procedimento descritto, si può ottenere una stima della distribuzione della perdita. Applicando il modello attuariale al contesto delle richieste di risarcimento, la severity dell'evento si può rappresentare con l'importo risarcito associato alla richiesta. Per calcolare la frequency, invece, si suddivide il periodo di tempo coperto dai dati in intervalli di ampiezza fissata e si conta il numero di richieste pervenute in ciascuno. Supponendo ad esempio che i dati a disposizione riguardino 
1. Estrazione di un valore di frequency

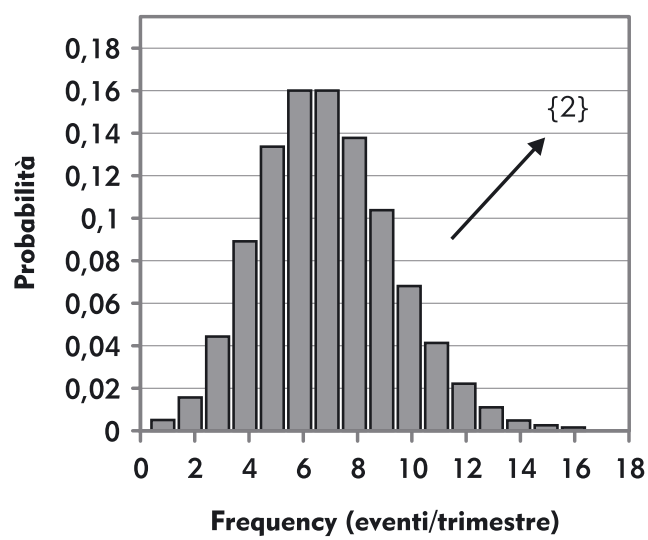

\section{Estrazione dei valori di severity}

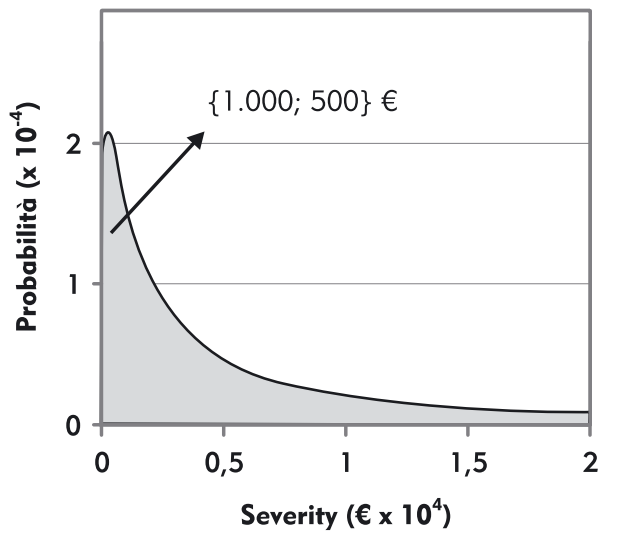

3. Somma dei valori di severity estratti<smiles>[3H][3H]</smiles>

Singolo scenario $\longrightarrow\{1.500\} €$

Figura 1. Esempio di calcolo di uno scenario applicando la simulazione Monte Carlo

8 anni si sceglie un intervallo di tempo minore, ad esempio 3 mesi, e si conta quante richieste di risarcimento sono pervenute all'azienda ospedaliera in ciascun trimestre. In questo modo per la severity si ha un numero di valori pari agli importi presenti, mentre per la frequency si hanno tanti valori quanti sono gli intervalli in cui è stato suddiviso l'orizzonte temporale coperto dai dati, cioè, nell'esempio, 32.

Per ottenere una stima della perdita utilizzando il modello attuariale occorre dunque avere a disposizione le distribuzioni di frequency e severity che descrivono l'andamento della frequenza e della gravità degli eventi. La stima di queste distribuzioni, solitamente ricavate tramite un approccio parametrico, è stata implementata in modo efficiente e in grado di tener conto delle caratteristiche dei dati a disposizione ricorrendo anche a tecniche di stima non parametrica [10]. Nel caso delle analisi stratificate, sono state impiegate delle tecniche avanzate di stima Bayesiana $[11,12]$ per tener conto anche del numero ridotto di dati a disposizione.

Dopo aver ricavato le distribuzioni di frequency e severity si applica la simulazione di tipo Monte Carlo per ottenere una stima della distribuzione della perdita da cui sono stati calcolati alcuni indici di rischio quantitativi tra cui il valore atteso e la mediana della distribuzione della perdita, il Value at risk e la perdita inattesa, pari alla differenza tra Var e valore atteso. Nelle analisi stratificate sono stati ricavati due indicatori aggiuntivi utili per poter meglio confrontare le diverse categorie. Il primo, pari al rapporto tra la perdita inattesa e il numero di letti di ciascuna classe, permette di confrontare i risultati ottenuti dall'analisi delle richieste di risarcimento con l'entità delle prestazioni erogate, ottenendo un confronto più equo tra le diverse categorie. Il secondo indicatore, rappresentato dal rapporto tra il Var e la mediana della distribuzione della perdita, cioè dal rapporto tra il $99^{\circ}$ e il $50^{\circ}$ percentile, può essere invece utilizzato per valutare l'andamento della perdita e gli eventi avversi associati.

\section{| DAT |}

Il metodo sviluppato è stato applicato ai dati riguardanti le richieste di risarcimento pervenute dal 1999 al primo semestre del 2007 all'Azienda Ospedaliera (A.O.) di Lodi, in Lombardia.

Il data set presenta 317 casi e 33 attributi che possono essere suddivisi in 3 categorie principali:

1. variabili relative alla richiesta di risarcimento, come Importo Risarcito, Stato del Sinistro, Data della Richiesta di Risarcimento, Data di Chiusura della Richiesta, ecc.;

2. informazioni riguardanti il sinistro, come Struttura Erogante, Area di Rischio, Data Evento, Descrizione Evento, ecc.;

3. codici identificativi, come Contratto Assicurativo, Identificativo Sinistro, ecc.

Tra le variabili relative alla richiesta di risarcimento, l'attributo Stato del Sinistro indica lo stato della richiesta di risarcimento e assume il valore Aperto se la richiesta è ancora in fase di valutazione, mentre è pari a Chiuso se è già stato stabilito un Importo Ri- 


\begin{tabular}{|lccc|}
$\begin{array}{c}\text { Risultati ottenuti } \\
\text { su } 10 \text { prove }\end{array}$ & $\begin{array}{c}\text { Media della } \\
\text { perdita }(\Theta)\end{array}$ & $\begin{array}{c}\text { Var } \\
\text { annuo }(\Theta)\end{array}$ & $\begin{array}{c}\text { Perdita } \\
\text { inattesa }(\Theta)\end{array}$ \\
\hline Media & 494.273 & 2.014 .070 & 1.519 .797 \\
\hline Deviazione standard & 12.779 & 136.415 & 128.418 \\
\hline
\end{tabular}

Tabella I. Risultati dell'analisi globale

sarcito. Nel data set in analisi sono presenti 110 casi aperti e 207 casi chiusi. I casi chiusi sono associati a un importo risarcito che può anche essere pari a zero, nel caso in cui la richiesta sia stata analizzata e chiusa, ma non accolta. Nell'applicazione del metodo descritto si è scelto di rappresentare la severity, cioè la gravità dell'evento, con l'Importo Risarcito, mentre la frequency è stata calcolata contando il numero di richieste di risarcimento pervenute all'A.O. in ciascun trimestre. La metodologia sviluppata è stata quindi applicata alle richieste di risarcimento chiuse e poiché manca un valore per l'attributo Importo Risarcito sono stati considerati 206 casi. Su questi rispetto all'attributo Importo Risarcito si hanno 63 valori pari a 0 , il massimo e la media sono di circa $315 \mathrm{~K} €$ e $13 \mathrm{~K} €$ mentre il $5^{\circ}$ e il $95^{\circ}$ percentile sono rispettivamente pari a 0 e a circa $73 \mathrm{~K} €$. Gli attributi riguardanti il sinistro oggetto della richiesta possono essere utilizzati per suddividere $\mathrm{i}$ dati in categorie di interesse esaminabili nelle analisi stratificate. Nel contesto in esame sono stati ritenuti di particolare interesse gli attributi Area di Rischio e Struttura Erogante. La prima variabile indica a quale specialità medica afferisce la prestazione, ad esempio l'area Chirurgica, indipendentemente dalla specifica struttura erogante, mentre la seconda identifica quale tra i 4 presidi ospedalieri e i diversi ambulatori gestiti dall'A.O. di Lodi ha fornito la prestazione oggetto della richiesta.

\section{RISULTATI}

La metodologia sviluppata è stata applicata sia al data set nel suo complesso, nell'analisi globale, sia ai dati suddivisi in gruppi, nelle analisi stratificate. Per entrambe le tipologie di analisi è stato utilizzato lo stesso numero di simulazioni per la stima della distribuzione della perdita con la tecnica della simulazione Monte Carlo e lo stesso orizzonte temporale per il calcolo della frequency. Dopo ricerche in letteratura [13] e prove sperimentali sono state scelte 15.000 simulazioni ed è stato ritenuto adeguato l'orizzonte trimestrale per il calcolo della frequency.

Per quanto concerne l'analisi globale, per ottenere risultati ancor più affidabili i valori degli indicatori sono stati ottenuti mediando i risultati di 10 esecuzioni. In questa analisi è stato applicato un fitting parametrico in cui sono risultate avere il miglior adattamento ai dati la distribuzione Binomiale Negativa per la frequency e quella Lognormale per la severity. Come si può notare in Tabella I, applicando la metodologia sviluppata al data set nel suo complesso è stato ricavato un valore atteso della perdita pari a circa $500 \mathrm{~K} €$, un Value at risk di circa 2 M€ e quindi un ammontare della perdita inattesa di circa 1,5 M€. Il valore del Var ricavato può essere considerato rappresentativo della massima perdita attesa in un anno ed è risultato compatibile con il premio assicurativo annuale pagato dall'azienda ospedaliera.

Per poter analizzare la rischiosità dell'azienda non solo nel suo insieme, ma anche rispetto a singole variabili di interesse, sono state effettuate due analisi stratificate considerando gli attributi Area di Rischio e Struttura Erogante. In queste analisi per la severity è stato applicato un metodo di identificazione parametrica, in cui la distribuzione Lognormale è risultata avere un miglior adattamento rispetto a quella Esponenziale nella quasi totalità dei casi. Per la frequency è stato utilizzato invece un modello Bayesiano, per ovviare alla riduzione dei valori significativi dovuta alla suddivisione dei dati in gruppi.

Come si può notare dalla Tabella II, che riporta $\mathrm{i}$ risultati ottenuti per alcune aree di rischio dell'A.O., l'area Ostetrica e Ginecologica presenta sia il Var sia il rapporto tra perdita inattesa e posti letto maggiori, risultando così la più rischiosa. Quest'area presenta anche un valore abbastanza elevato per il rapporto tra Var e mediana, che permette di intuire l'andamento della perdita. Un elevato valore di questo indicatore, infatti, significa che il $99^{\circ}$ percentile risulta molto scostato rispetto al $50^{\circ}$ e quindi indica che la distribuzione della perdita è caratterizzata da eventi rari, ma catastrofici. Questo fenomeno si può osservare anche dal grafico della distribuzione della perdita, in Figura

\begin{tabular}{|lcccc|}
\multicolumn{1}{c}{ Area di Rischio } & Var annuo $(\Theta)$ & $\begin{array}{c}\text { Mediana } \\
\text { della perdita }(\Theta)\end{array}$ & $\begin{array}{c}\text { Perdita inattesa/ } \\
\text { posti letto }(€)\end{array}$ & $\begin{array}{c}\text { Var/mediana } \\
\text { della perdita }\end{array}$ \\
\hline Ostetrica e ginecologica & 1.691 .000 & 42.658 & 22.027 & 40 \\
\hline Chirurgica & 1.129 .700 & 134.030 & 3.622 & 8 \\
\hline Medica & 689.010 & 32.353 & 1.310 & 21 \\
\hline
\end{tabular}

Tabella II. Risultati dell'analisi stratificata rispetto all'attributo Area di Rischio (estratto) 


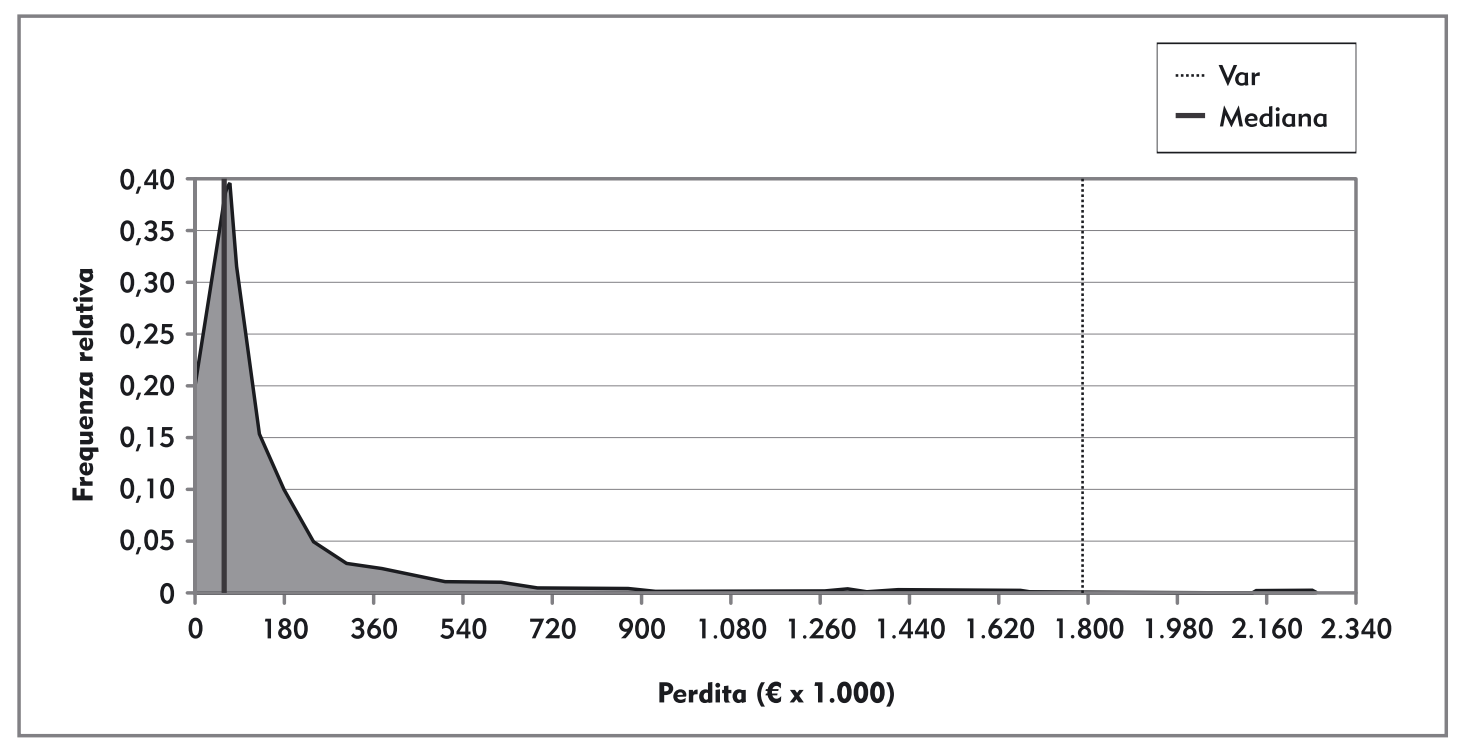

Figura 2. Distribuzione della perdita per l'Area di Rischio Ostetrica e Ginecologica

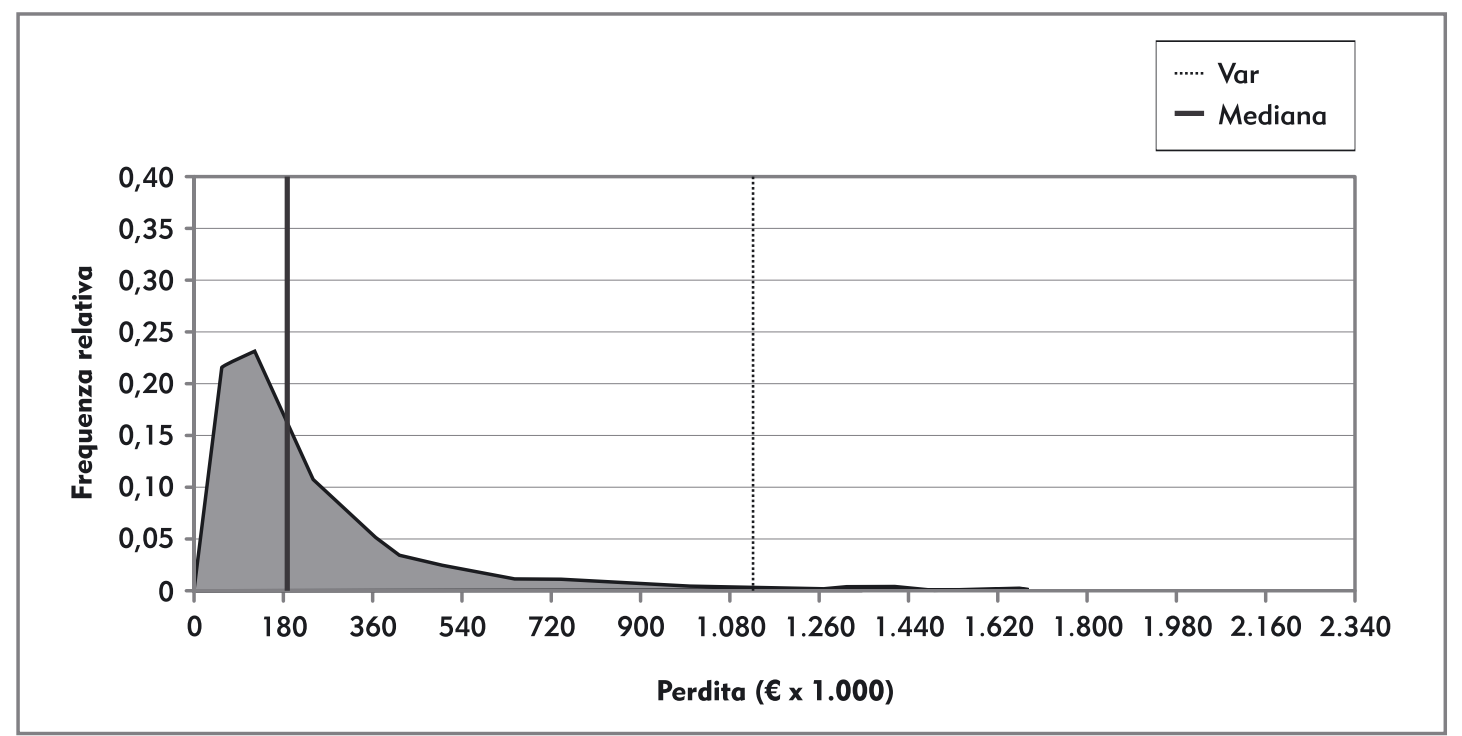

Figura 3. Distribuzione della perdita per l'Area di Rischio Chirurgica

2 , che presenta la maggior parte dei valori concentrati nella parte sinistra, corrispondente a importi relativamente bassi, ma riporta anche una lunga e bassa coda sulla destra che mostra pochi eventi associati a importi molto elevati. Aree con un basso rapporto tra Var e mediana, come ad esempio quella Chirurgica, sono associate invece a risarcimenti maggiormente uniformi e mediamente più alti, come si può notare in Figura 3. Il rapporto tra Var e mediana può dunque essere utile per caratterizzare l'andamento della perdita di ogni categoria e la tipologia di eventi avversi associati.

Riguardo all'analisi stratificata rispetto ai valori dell'attributo Struttura Erogante si può notare in Tabella III che l'Ospedale 3 risulta avere un Var di poco inferiore rispetto all'Ospedale 1, ma presenta di gran lunga il maggior rapporto tra perdita inattesa e numero di posti letto e per questo può

\begin{tabular}{|lcccc|}
\hline $\begin{array}{c}\text { Struttura } \\
\text { Erogante }\end{array}$ & $\begin{array}{c}\text { Var } \\
\text { annuo }(€)\end{array}$ & $\begin{array}{c}\text { Mediana } \\
\text { della } \\
\text { perdita }(€)\end{array}$ & $\begin{array}{c}\text { Perdita inat- } \\
\text { tesa/ posti } \\
\text { letto }(€)\end{array}$ & $\begin{array}{c}\text { Var/ } \\
\text { mediana } \\
\text { della perdita }\end{array}$ \\
\hline Ospedale 1 & 1.412 .000 & 178.240 & 2.615 & 8 \\
\hline Ospedale 3 & 1.334 .300 & 52.800 & 12.517 & 25 \\
\hline Ospedale 4 & 441.830 & 25.157 & 1.872 & 18 \\
\hline Ospedale 2 & 122.860 & 29.746 & 467 & 4 \\
\hline
\end{tabular}

Tabella III. Risultati dell'analisi stratificata rispetto all'attributo Struttura Erogante (estratto)

necessitare di azioni di miglioramento. Lalto valore del rapporto tra Var e mediana e il grafico della distribuzione della perdita, in Figura 4, mostrano che questa struttura è caratterizzata da perdite 


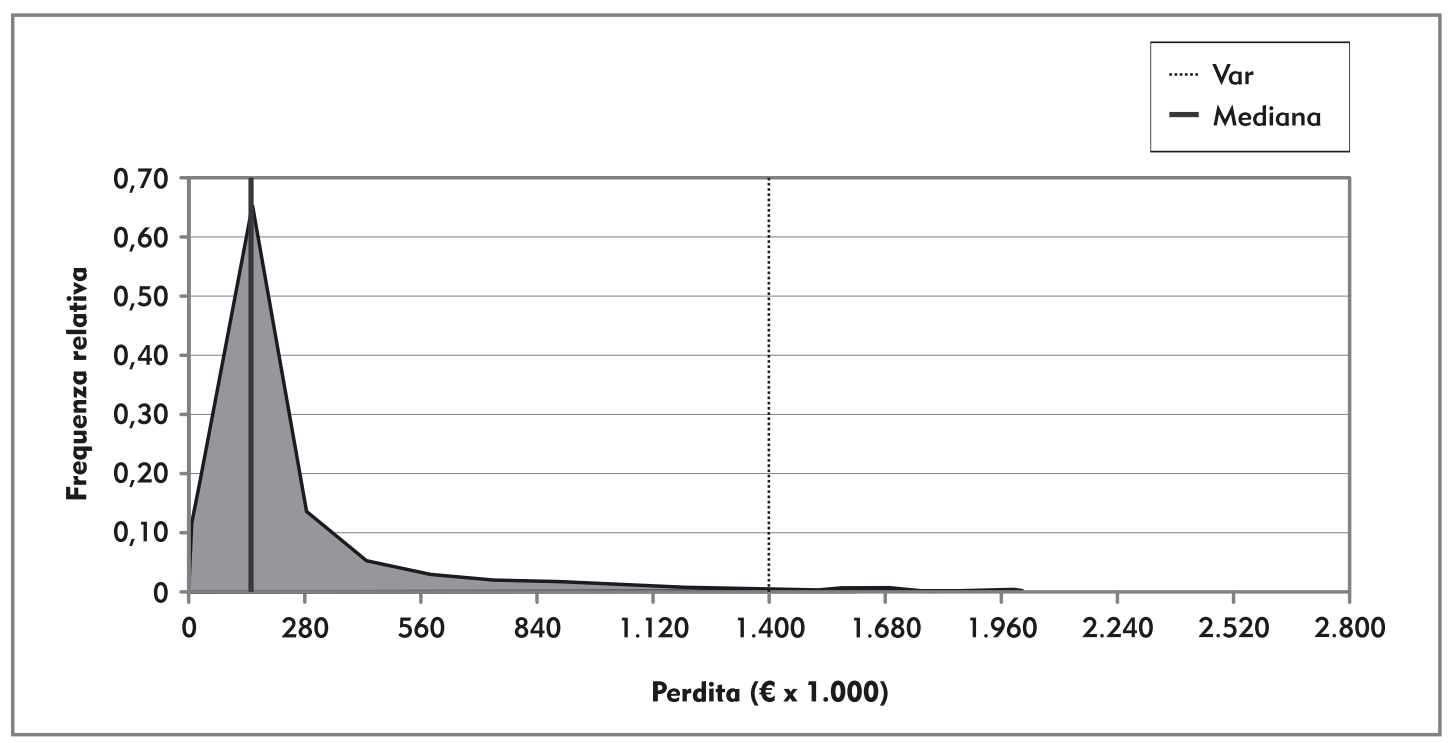

Figura 4. Distribuzione della perdita per la Struttura Erogante Ospedale 3

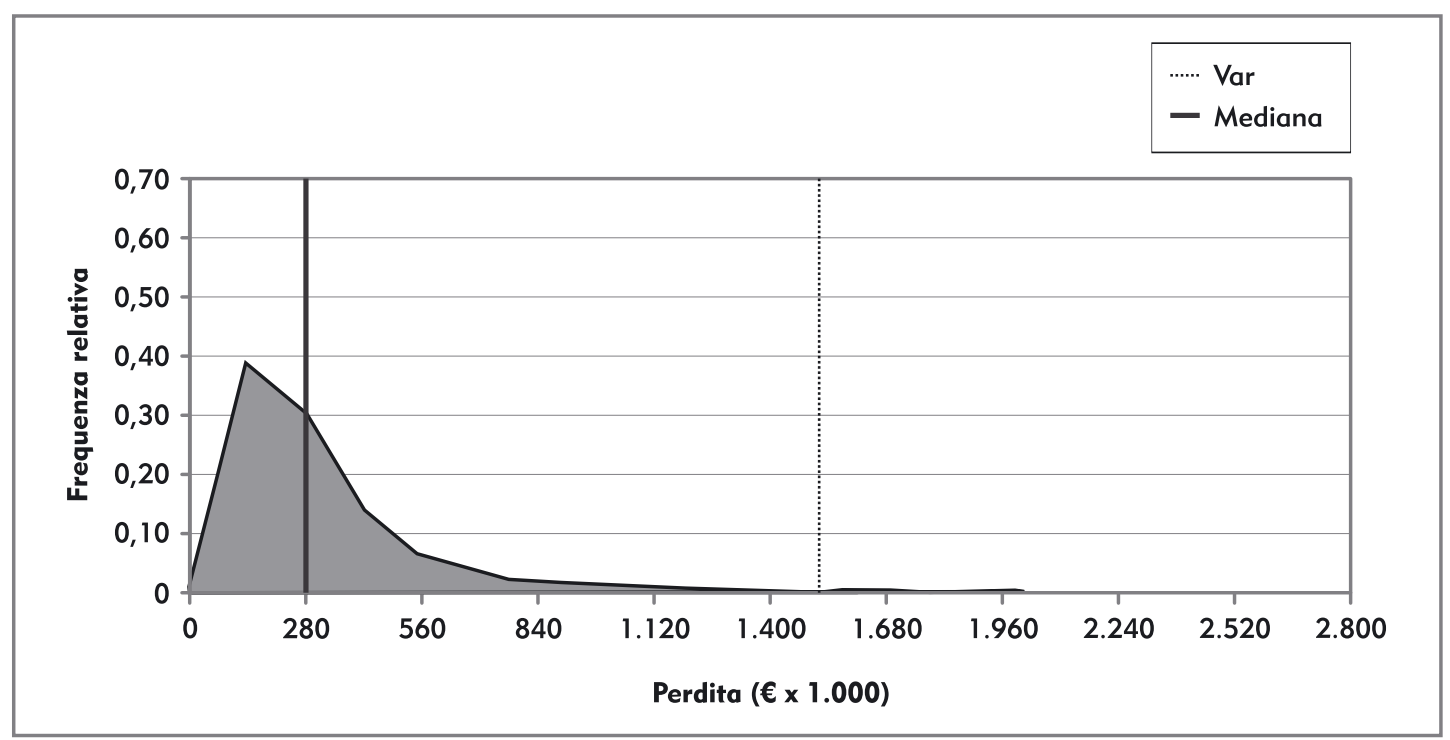

Figura 5. Distribuzione della perdita per la Struttura Erogante Ospedale 1

prevalentemente basse, ma anche da pochi casi relativi a risarcimenti molto elevati; fenomeno che può essere causato da una cattiva gestione delle eccezioni. L'Ospedale 1 invece, come si può osservare in Figura 5, è associato a incidenti con valori maggiormente distribuiti e mediamente più alti $\mathrm{e}$ quindi si può pensare che sia affetto da problemi di gestione o errori sistematici.

\section{CONCLUSIONI}

In questo articolo è stato proposto un metodo probabilistico per analizzare i dati relativi alle ri- chieste di risarcimento pervenute alle aziende ospedaliere e ricavarne indici di rischio quantitativi. Il metodo è basato sul modello attuariale, largamente diffuso nei settori bancario e finanziario per calcolare il rischio operativo, ma utilizza anche diverse tecniche, tra cui modelli gerarchici Bayesiani e metodi di fitting non parametrico, per ottenere risultati significativi anche a fronte di data set di modeste dimensioni.

La metodologia sviluppata ha permesso di analizzare 206 casi di richieste di risarcimento distribuite su un orizzonte temporale di 8 anni valutandole sia nel loro complesso, sia suddivise rispetto ai valori di variabili di interesse. I risultati ottenuti nell'analisi globale possono essere sfruttati dall'azienda ospedaliera per contrattare in maniera maggiormente consapevole il premio assicura- 
tivo prevedendo, per esempio, una franchigia pari al valore atteso della perdita e assicurandosi solo per la perdita inattesa. In questo modo l'A.O. pagherebbe direttamente i risarcimenti fino al raggiungimento della franchigia, ma otterrebbe sia una riduzione del premio assicurativo, sia un vantaggio diretto a seguito dell'introduzione di interventi di miglioramento volti alla riduzione dei risarcimenti associati ai sinistri. I risultati delle analisi stratificate possono invece essere sfruttati per identificare le maggiori sorgenti di rischio, poiché consentono di analizzare e confrontare la rischiosità di ciascuna categoria di interesse.

Il metodo proposto può dunque rivelarsi un utile strumento per sfruttare i dati relativi alle richieste di risarcimento, già disponibili a differenza di quelli relativi agli errori clinici, per ricavare indici oggettivi quantificabili anche in termini monetari. Valutando questi indicatori l'azienda ospedaliera può conoscere meglio la propria sinistrosità, e una maggiore consapevolezza della propria rischiosità a livello sia dell'intera struttura, sia dei singoli settori è il primo passo verso la definizione e l'applicazione di azioni volte alla prevenzione e riduzione degli eventi avversi.

\section{DISCLOSURE}

Gli Autori dichiarano di non avere alcun confitto di interessi di natura finanziaria.

\section{BIBLIOGRAFIA}

1. Ministero della Salute. Rapporto sulla $1^{\circ}$ rilevazione nazionale relativa agli "aspetti assicurativi in ambito di gestione aziendale del rischio clinico". Dipartimento della Qualità [Internet]. 2006 September [cited 2010 Janaury 4]. Available from: http://www.ministerosalute.it/imgs/C_17_pubblicazioni_544_allegato.pdf

2. Cruz MG. Modeling, measurement and hedging operational risk. Chichester: John Wiley \& Sons Ltd, 2002

3. Frachot A, Moudoulaud O, Roncalli T. Loss Distribution Approach in Practice. Technical report, Groupe de Recherche Operationnelle, Crédit Lyonnais [Internet]. 2003 May [cited 2010 Janaury 4]. Available from: http:// www.maths-fi.com/malliavin/Loss_Distribution_Approach_in_Practice_05_02_2003.pdf

4. Alexander C. Operational risk: regulation, analysis and management. London: Prentice Hall, Financial Times, 2003

5. Jorion P. Value at risk: the new benchmark for managing financial risk. New York: McGraw-Hill, 2000

6. King JL. Operational Risk. Measurement and modelling. $2^{\text {nd }}$ edition. Chichester: John Wiley \& Sons Ltd, 2001

7. Vose D. Risk Analysis. A Quantitative guide. $2^{\text {nd }}$ edition, Chichester: John Wiley \& Sons Ltd, 2000

8. Robert CP, Casella G. Monte Carlo statistical methods. New York: Springer Science Business Media Inc, 2004

9. Fishmann GS. Monte Carlo: Concepts, Algorithms and Applications. New York: Springer - Verlag New York Inc, 1996

10. Hastie T, Tibshirani R, Friedman J. The Elements of Statistical Computing - Data Mining. Inference and Prediction. New York: Springer, 2001

11. Bellazzi R, Riva A. Learning Bayesian network probabilities from longitudinal data. IEEE Trans 1998; 28: 629-36

12. Gelman A, Carlin, JB, Stern HS, Rubin DB. Bayesian Data Analysis. London: Chapman \& Hall, 1995

13. Fanoni F, Giudici P, Muratori GM. Il rischio operativo - Monitoraggio, misurazione, mitigazione. Milano: Il Sole 24 Ore, 2005

\section{CORRESPONDING AUTHOR}

Dott.ssa Simona Delle Caronare; e-mail: simona.dallecarbonare@gmail.com 